Supplement of Web Ecol., 17, 19-27, 2017

http://www.web-ecol.net/17/19/2017/

doi:10.5194/we-17-19-2017-supplement

(C) Author(s) 2017. CC Attribution 3.0 License.

(c) (i)

Supplement of

\title{
Sand quarry wetlands provide high-quality habitat for native amphibians
}

Michael Sievers

Correspondence to: Michael Sievers (msievers@student.unimelb.edu.au)

The copyright of individual parts of the supplement might differ from the CC-BY 3.0 licence. 
Section S1. Raw data for the calculation and analysis of levels of

developmental instability within quarry and reference wetlands. Treat: quarry (Q) and reference (R) wetlands, Ind: individual number, Sp: amphibian species, Rep: replicate measurement number, SVL: snout-vent length, Side: side measured, and the length of the forelimb and hindlimb (as Alford et al 2007).

$\begin{array}{llllllll}\text { Treat } & \text { Ind } & \text { Sp } & \text { Rep } & \text { SVL } & \text { Side } & \text { Forelimb } & \text { Hindlimb } \\ \text { Q } & 1 & \text { Crinia } & 1 & 22.2 & \mathrm{~L} & 6.22 & 8.03 \\ \text { Q } & 1 & \text { Crinia } & 1 & 22.2 & \mathrm{R} & 6.34 & 8.35 \\ \mathrm{Q} & 1 & \text { Crinia } & 2 & 22.2 & \mathrm{~L} & 6.36 & 8.14 \\ \mathrm{Q} & 1 & \text { Crinia } & 2 & 22.2 & \mathrm{R} & 6.37 & 8.22 \\ \mathrm{Q} & 1 & \text { Crinia } & 3 & 22.2 & \mathrm{~L} & 6.23 & 7.99 \\ \mathrm{Q} & 1 & \text { Crinia } & 3 & 22.2 & \mathrm{R} & 6.21 & 8.17 \\ \mathrm{Q} & 2 & \text { Crinia } & 1 & 19.5 & \mathrm{~L} & 5.96 & 7.74 \\ \mathrm{Q} & 2 & \text { Crinia } & 1 & 19.5 & \mathrm{R} & 5.84 & 7.96 \\ \mathrm{Q} & 2 & \text { Crinia } & 2 & 19.5 & \mathrm{~L} & 5.83 & 7.77 \\ \mathrm{Q} & 2 & \text { Crinia } & 2 & 19.5 & \mathrm{R} & 5.77 & 7.71 \\ \mathrm{Q} & 2 & \text { Crinia } & 3 & 19.5 & \mathrm{~L} & 5.85 & 7.7 \\ \mathrm{Q} & 2 & \text { Crinia } & 3 & 19.5 & \mathrm{R} & 5.81 & 7.66 \\ \mathrm{Q} & 3 & \text { Crinia } & 1 & 18.4 & \mathrm{~L} & 5.58 & 7.71 \\ \mathrm{Q} & 3 & \text { Crinia } & 1 & 18.4 & \mathrm{R} & 5.52 & 7.5 \\ \mathrm{Q} & 3 & \text { Crinia } & 2 & 18.4 & \mathrm{~L} & 5.34 & 7.56 \\ \mathrm{Q} & 3 & \text { Crinia } & 2 & 18.4 & \mathrm{R} & 5.55 & 7.49 \\ \mathrm{Q} & 3 & \text { Crinia } & 3 & 18.4 & \mathrm{~L} & 5.42 & 7.65 \\ \mathrm{Q} & 3 & \text { Crinia } & 3 & 18.4 & \mathrm{R} & 5.5 & 7.91 \\ \mathrm{Q} & 4 & \text { Crinia } & 1 & 20.9 & \mathrm{~L} & 6 & 8.11 \\ \mathrm{Q} & 4 & \text { Crinia } & 1 & 20.9 & \mathrm{R} & 6.08 & 8.3 \\ \mathrm{Q} & 4 & \text { Crinia } & 2 & 20.9 & \mathrm{~L} & 6.05 & 8.2 \\ \mathrm{Q} & 4 & \text { Crinia } & 2 & 20.9 & \mathrm{R} & 6.01 & 8.3 \\ \mathrm{Q} & 4 & \text { Crinia } & 3 & 20.9 & \mathrm{~L} & 6.1 & 8 \\ \mathrm{Q} & 4 & \text { Crinia } & 3 & 20.9 & \mathrm{R} & 6.1 & 8.25 \\ \mathrm{Q} & 5 & \text { Ewingii } & 1 & 25.6 & \mathrm{~L} & 10.1 & 12.55 \\ \mathrm{Q} & 5 & \text { Ewingii } & 1 & 25.6 & \mathrm{R} & 10.2 & 12.7 \\ \mathrm{Q} & 5 & \text { Ewingii } & 2 & 25.6 & \mathrm{~L} & 10.28 & 12.48 \\ \mathrm{Q} & 5 & \text { Ewingii } & 2 & 25.6 & \mathrm{R} & 10.05 & 12.68 \\ \mathrm{Q} & 5 & \text { Ewingii } & 3 & 25.6 & \mathrm{~L} & 10.3 & 12.6 \\ \mathrm{Q} & 5 & \text { Ewingii } & 3 & 25.6 & \mathrm{R} & 10.11 & 12.66 \\ \mathrm{Q} & 6 & \text { Limno } \mathrm{p} & 1 & 36.6 & \mathrm{~L} & 12.5 & 16.64 \\ \mathrm{Q} & 6 & \text { Limno } \mathrm{p} & 1 & 36.6 & \mathrm{R} & 12.37 & 16.55 \\ \mathrm{Q} & 6 & \text { Limno } \mathrm{p} & 2 & 36.6 & \mathrm{~L} & 12.66 & 16.52 \\ & & & & & & & \end{array}$




\begin{tabular}{|c|c|c|c|c|c|c|c|}
\hline$Q$ & 6 & Limno $p$ & 2 & 36.6 & $\mathrm{R}$ & 12.4 & 16.51 \\
\hline $\mathrm{Q}$ & 6 & Limno $p$ & 3 & 36.6 & $\mathrm{~L}$ & 12.69 & 16.6 \\
\hline $\mathrm{Q}$ & 6 & Limno $p$ & 3 & 36.6 & $\mathrm{R}$ & 12.33 & 16.6 \\
\hline $\mathrm{R}$ & 7 & Crinia & 1 & 20.5 & $\mathrm{~L}$ & 6.24 & 8.42 \\
\hline $\mathrm{R}$ & 7 & Crinia & 1 & 20.5 & $\mathrm{R}$ & 5.92 & 8.55 \\
\hline $\mathrm{R}$ & 7 & Crinia & 2 & 20.5 & $\mathrm{~L}$ & 6.18 & 8.35 \\
\hline $\mathrm{R}$ & 7 & Crinia & 2 & 20.5 & $\mathrm{R}$ & 5.89 & 8.61 \\
\hline $\mathrm{R}$ & 7 & Crinia & 3 & 20.5 & $\mathrm{~L}$ & 6.15 & 8.3 \\
\hline $\mathrm{R}$ & 7 & Crinia & 3 & 20.5 & $\mathrm{R}$ & 5.96 & 8.5 \\
\hline Q & 8 & Crinia & 1 & 15.5 & $\mathrm{~L}$ & 5.5 & 7.2 \\
\hline Q & 8 & Crinia & 1 & 15.5 & $\mathrm{R}$ & 5.49 & 7.16 \\
\hline $\mathrm{Q}$ & 8 & Crinia & 2 & 15.5 & $\mathrm{~L}$ & 5.71 & 7.24 \\
\hline Q & 8 & Crinia & 2 & 15.5 & $\mathrm{R}$ & 5.49 & 7.3 \\
\hline Q & 8 & Crinia & 3 & 15.5 & $\mathrm{~L}$ & 5.66 & 7.28 \\
\hline $\mathrm{Q}$ & 8 & Crinia & 3 & 15.5 & $\mathrm{R}$ & 5.58 & 7.18 \\
\hline $\mathrm{Q}$ & 9 & Crinia & 1 & 19.9 & $\mathrm{~L}$ & 6.08 & 8.23 \\
\hline Q & 9 & Crinia & 1 & 19.9 & $\mathrm{R}$ & 6.18 & 8.22 \\
\hline $\mathrm{Q}$ & 9 & Crinia & 2 & 19.9 & $\mathrm{~L}$ & 6.15 & 8.34 \\
\hline $\mathrm{Q}$ & 9 & Crinia & 2 & 19.9 & $\mathrm{R}$ & 6.2 & 8.35 \\
\hline $\mathrm{Q}$ & 9 & Crinia & 3 & 19.9 & $\mathrm{~L}$ & 6.2 & 8.15 \\
\hline Q & 9 & Crinia & 3 & 19.9 & $\mathrm{R}$ & 6.07 & 8.4 \\
\hline $\mathrm{Q}$ & 10 & Crinia & 1 & 24.1 & $\mathrm{~L}$ & 6.88 & 9.9 \\
\hline Q & 10 & Crinia & 1 & 24.1 & $\mathrm{R}$ & 6.79 & 9.8 \\
\hline Q & 10 & Crinia & 2 & 24.1 & $\mathrm{~L}$ & 6.81 & 9.84 \\
\hline $\mathrm{Q}$ & 10 & Crinia & 2 & 24.1 & $\mathrm{R}$ & 6.89 & 9.88 \\
\hline Q & 10 & Crinia & 3 & 24.1 & $\mathrm{~L}$ & 6.93 & 9.95 \\
\hline Q & 10 & Crinia & 3 & 24.1 & $\mathrm{R}$ & 6.88 & 9.9 \\
\hline Q & 11 & Crinia & 1 & 19.8 & $\mathrm{~L}$ & 5.99 & 8.46 \\
\hline $\mathrm{Q}$ & 11 & Crinia & 1 & 19.8 & $\mathrm{R}$ & 6.11 & 8.34 \\
\hline Q & 11 & Crinia & 2 & 19.8 & $\mathrm{~L}$ & 6.09 & 8.48 \\
\hline Q & 11 & Crinia & 2 & 19.8 & $\mathrm{R}$ & 6.09 & 8.42 \\
\hline Q & 11 & Crinia & 3 & 19.8 & $\mathrm{~L}$ & 5.97 & 8.44 \\
\hline Q & 11 & Crinia & 3 & 19.8 & $\mathrm{R}$ & 6.16 & 8.34 \\
\hline $\mathrm{Q}$ & 12 & Limno $p$ & 1 & 29.6 & $\mathrm{~L}$ & 11.33 & 15.99 \\
\hline Q & 12 & Limno $p$ & 1 & 29.6 & $\mathrm{R}$ & 11.36 & 15.8 \\
\hline $\mathrm{Q}$ & 12 & Limno $p$ & 2 & 29.6 & $\mathrm{~L}$ & 11.32 & 15.9 \\
\hline Q & 12 & Limno $\mathrm{p}$ & 2 & 29.6 & $\mathrm{R}$ & 11.44 & 15.89 \\
\hline $\mathrm{Q}$ & 12 & Limno $p$ & 3 & 29.6 & $\mathrm{~L}$ & 11.3 & 15.87 \\
\hline Q & 12 & Limno $p$ & 3 & 29.6 & $\mathrm{R}$ & 11.44 & 15.73 \\
\hline Q & 13 & Crinia & 1 & 23.4 & $\mathrm{~L}$ & 6.66 & 8.22 \\
\hline Q & 13 & Crinia & 1 & 23.4 & $\mathrm{R}$ & 6.52 & 8.3 \\
\hline $\mathrm{Q}$ & 13 & Crinia & 2 & 23.4 & $\mathrm{~L}$ & 6.61 & 8.19 \\
\hline $\mathrm{Q}$ & 13 & Crinia & 2 & 23.4 & $\mathrm{R}$ & 6.59 & 8.22 \\
\hline $\mathrm{Q}$ & 13 & Crinia & 3 & 23.4 & $\mathrm{~L}$ & 6.61 & 8.3 \\
\hline $\mathrm{Q}$ & 13 & Crinia & 3 & 23.4 & $\mathrm{R}$ & 6.59 & 8.23 \\
\hline $\mathrm{Q}$ & 14 & Crinia & 1 & 20.8 & $\mathrm{~L}$ & 6.4 & 7.88 \\
\hline
\end{tabular}




$\begin{array}{llllllll}\mathrm{Q} & 14 & \text { Crinia } & 1 & 20.8 & \mathrm{R} & 6.29 & 7.9 \\ \mathrm{Q} & 14 & \text { Crinia } & 2 & 20.8 & \mathrm{~L} & 6.44 & 7.8 \\ \mathrm{Q} & 14 & \text { Crinia } & 2 & 20.8 & \mathrm{R} & 6.29 & 7.99 \\ \mathrm{Q} & 14 & \text { Crinia } & 3 & 20.8 & \mathrm{~L} & 6.37 & 7.89 \\ \mathrm{Q} & 14 & \text { Crinia } & 3 & 20.8 & \mathrm{R} & 6.29 & 8 \\ \mathrm{R} & 15 & \text { Crinia } & 1 & 22.2 & \mathrm{~L} & 6.66 & 8.01 \\ \mathrm{R} & 15 & \text { Crinia } & 1 & 22.2 & \mathrm{R} & 6.5 & 8.22 \\ \mathrm{R} & 15 & \text { Crinia } & 2 & 22.2 & \mathrm{~L} & 6.7 & 7.99 \\ \mathrm{R} & 15 & \text { Crinia } & 2 & 22.2 & \mathrm{R} & 6.42 & 8.33 \\ \mathrm{R} & 15 & \text { Crinia } & 3 & 22.2 & \mathrm{~L} & 6.66 & 8 \\ \mathrm{R} & 15 & \text { Crinia } & 3 & 22.2 & \mathrm{R} & 6.44 & 8.26 \\ \mathrm{R} & 16 & \text { Crinia } & 1 & 18.7 & \mathrm{~L} & 5.9 & 7.72 \\ \mathrm{R} & 16 & \text { Crinia } & 1 & 18.7 & \mathrm{R} & 5.8 & 7.51 \\ \mathrm{R} & 16 & \text { Crinia } & 2 & 18.7 & \mathrm{~L} & 5.84 & 7.79 \\ \mathrm{R} & 16 & \text { Crinia } & 2 & 18.7 & \mathrm{R} & 5.77 & 7.55 \\ \mathrm{R} & 16 & \text { Crinia } & 3 & 18.7 & \mathrm{~L} & 5.8 & 7.77 \\ \mathrm{R} & 16 & \text { Crinia } & 3 & 18.7 & \mathrm{R} & 5.78 & 7.59 \\ \mathrm{R} & 17 & \text { Crinia } & 1 & 19 & \mathrm{~L} & 6.04 & 8.11 \\ \mathrm{R} & 17 & \text { Crinia } & 1 & 19 & \mathrm{R} & 5.8 & 8.33 \\ \mathrm{R} & 17 & \text { Crinia } & 2 & 19 & \mathrm{~L} & 5.99 & 8.16 \\ \mathrm{R} & 17 & \text { Crinia } & 2 & 19 & \mathrm{R} & 5.79 & 8.34 \\ \mathrm{R} & 17 & \text { Crinia } & 3 & 19 & \mathrm{~L} & 5.89 & 8.2 \\ \mathrm{R} & 17 & \text { Crinia } & 3 & 19 & \mathrm{R} & 5.83 & 8.3 \\ \mathrm{R} & 18 & \text { Crinia } & 1 & 21.1 & \mathrm{~L} & 6 & 8.33 \\ \mathrm{R} & 18 & \text { Crinia } & 1 & 21.1 & \mathrm{R} & 6.18 & 8.19 \\ \mathrm{R} & 18 & \text { Crinia } & 2 & 21.1 & \mathrm{~L} & 6.01 & 8.4 \\ \mathrm{R} & 18 & \text { Crinia } & 2 & 21.1 & \mathrm{R} & 6.17 & 8.17 \\ \mathrm{R} & 18 & \text { Crinia } & 3 & 21.1 & \mathrm{~L} & 6.1 & 8.36 \\ \mathrm{R} & 18 & \text { Crinia } & 3 & 21.1 & \mathrm{R} & 6.23 & 8.16 \\ \mathrm{R} & 19 & \text { Crinia } & 1 & 26.1 & \mathrm{~L} & 7.05 & 10.1 \\ \mathrm{R} & 19 & \text { Crinia } & 1 & 26.1 & \mathrm{R} & 7.3 & 9.88 \\ \mathrm{R} & 19 & \text { Crinia } & 2 & 26.1 & \mathrm{~L} & 7.11 & 10.19 \\ \mathrm{R} & 19 & \text { Crinia } & 2 & 26.1 & \mathrm{R} & 7.33 & 9.81 \\ \mathrm{R} & 19 & \text { Crinia } & 3 & 26.1 & \mathrm{~L} & 7.12 & 10.23 \\ \mathrm{R} & 19 & \text { Crinia } & 3 & 26.1 & \mathrm{R} & 7.24 & 9.9\end{array}$


Supplement Table S1. ANOVA for determining the presence of directional asymmetry and fluctuating asymmetry plus antisymmetry for both forelimbs and hindlimbs, and for comparing levels of devlopmental instability in the Common Eastern Froglet (Crinia signifera) from quarry and reference wetlands (SAMPLE). A significant p-value for directional asymmetry indicates that the degree of asymmetry cannot be used as an index of DI, as the ideal degree of symmetry cannot be known (i.e. structures appear to not be ideally bilateral). A significant p-value for FA + antisymmetry indicates that measurement error is small relative to the levels of fluctuating asymmetry and antisymmetry in the data, and thus, levels of developmental instability can be compared between populations. A significant p-value for quarry $v s$ reference wetlands indicatesthat levels of developmental instability are greater in one population. SIDE: left or right limbs; REP: replicate measurement number; IND: individual frog number.

\begin{tabular}{lccc}
\hline & & Forelimb & Hindlimb \\
\cline { 3 - 4 } Factor & df & \multicolumn{2}{c}{ Mean Square } \\
\hline SIDE & 1 & 0.023 & 0.013 \\
REP & 2 & 0.000 & 0.002 \\
SIDE * REP & 2 & 0.000 & 0.001 \\
IND & 15 & 1.233 & 3.199 \\
SIDE * IND & 15 & 0.025 & 0.041 \\
REP * IND & 30 & 0.003 & 0.006 \\
SIDE * REP * IND & 30 & 0.003 & 0.006 \\
\hline Directional asymmetry $\left(\mathrm{MS}_{\mathrm{SIDE}} / \mathrm{MS}_{\mathrm{SIDE}} * \mathrm{ND}\right)$ & & $\mathrm{F}_{1,15}=0.93, \mathrm{p}=0.35$ & $\mathrm{~F}_{1,15}=0.33, \mathrm{p}=0.58$
\end{tabular}




\begin{tabular}{|c|c|c|c|}
\hline $\mathrm{FA}+$ antisymmetry $\left(\mathrm{MS}_{\mathrm{SIDE}^{*} \mathrm{IND}} / \mathrm{MS}_{\mathrm{SIDE}^{*} \mathrm{IND}^{*} \mathrm{REP}}\right)$ & & $\mathrm{F}_{15,30}=7.38, \mathbf{p}<\mathbf{0 . 0 0 1}$ & $\mathrm{F}_{15,30}=7.14, \mathbf{p}<\mathbf{0 . 0 0 1}$ \\
\hline SAMPLE & 1 & 0.475 & 1.9309 \\
\hline SIDE & 1 & 0.023 & 0.0133 \\
\hline REP & 2 & 0.000 & 0.0016 \\
\hline IND & 9 & 1.582 & 4.3619 \\
\hline SAMPLE * SIDE & 1 & 0.013 & 0.019 \\
\hline SAMPLE * REP & 2 & 0.001 & 0.0008 \\
\hline SIDE * REP & 2 & 0.000 & 0.0007 \\
\hline SAMPLE * IND & 5 & 0.756 & 1.3601 \\
\hline SIDE * IND & 9 & 0.019 & 0.0469 \\
\hline REP * IND & 18 & 0.004 & 0.0067 \\
\hline $\operatorname{SIDE} * \mathrm{REP} * \mathrm{IND}$ & 2 & 0.001 & 0.0018 \\
\hline SAMPLE $*$ IND $*$ SIDE & 5 & 0.038 & 0.0341 \\
\hline SAMPLE * IND * REP & 10 & 0.003 & 0.0069 \\
\hline $\operatorname{SIDE} * \operatorname{REP} * \operatorname{IND}$ & 18 & 0.004 & 0.0046 \\
\hline SAMPLE $*$ IND * SIDE * REP & 10 & 0.003 & 0.0083 \\
\hline Quarry vs Reference (MS SAMPLE*IND*SIDE $/$ MS $_{\text {SAMPLE*IND*SIDE*REP }^{*}}$ & & $\mathrm{~F}_{5,10}=11.78, \mathbf{p}<\mathbf{0 . 0 0 1}$ & $\mathrm{F}_{5,10}=4.11, \mathbf{p}=\mathbf{0 . 0 2 7}$ \\
\hline
\end{tabular}




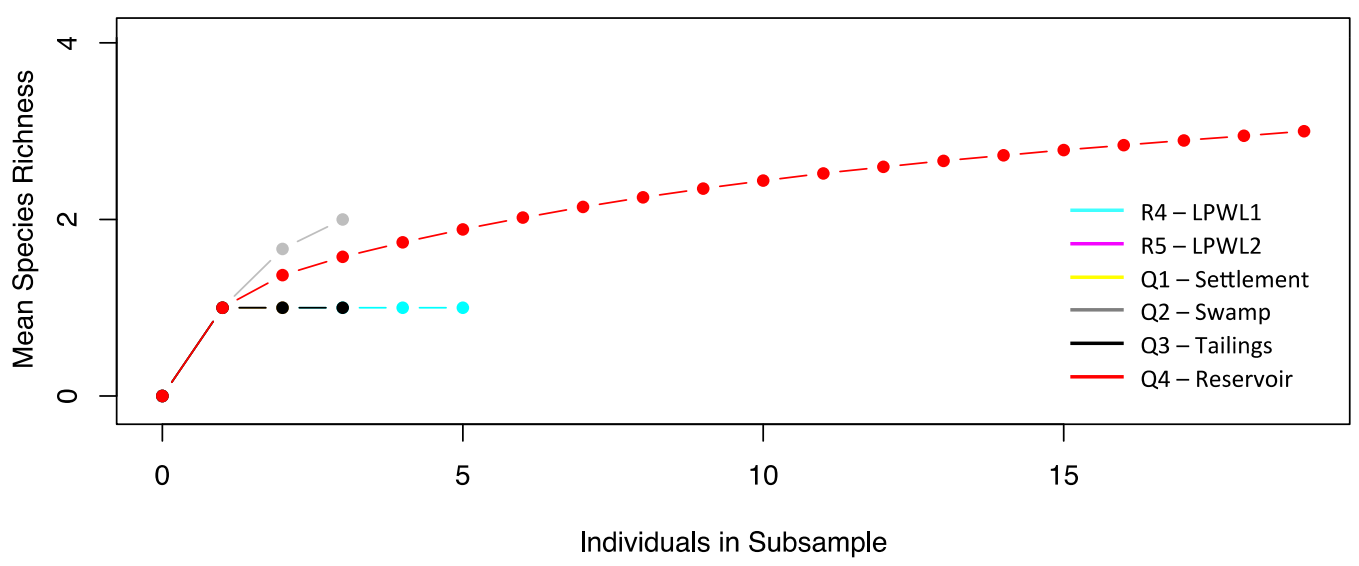

Supplement Figure S1. Rarefaction curve for the mean species richness in relation to the number of individuals in each subsample. See Table 1 caption for definition of wetland names. 